\title{
THE INFLUENCE OF ELLIPSOIDAL CORRECTIONS ON THE DETERMINATION OF THE ELEMENTS OF THE ANOMALOUS GRAVITATIONAL FIELD OF THE EARTH
}

\author{
Andrii Sohor ${ }^{1}$ \\ DOI: https://doi.org/10.30525/978-9934-588-38-9-60
}

Abstract. The content of the spherical approximation is to map the
points of the ellipsoid with geodetic coordinates to points on the sphere, the
spherical coordinates of which are taken as coordinates of the points on the
ellipsoid. Moreover, the values of the first order and the members of higher
orders are neglected. The described transformation of geodetic coordinates
into spherical coordinates is not the only way of establishing correspond-
ence between the reference ellipsoid and the auxiliary sphere. You can also
compare the spherical coordinates to the ellipsoidal coordinates in the form
of the given latitude and longitude. The use of the latter leads to simpler
formulas, but the use of geodetic coordinates has greater advantages from
a practical point of view, since it is difficult to convert the geodetic coordinates into the given ones for a large number of points.

It is necessary to find the same reflection by numerically comparing the geodetic coordinates on the ellipsoid and sphere, but with the preservation of the values of the first order. Therefore, it is logical to say that ellipsoidal corrections should be considered in order to obtain higher accuracy in calculating the components of the Earth's anomalous gravitational field.

The methodology of such a scientific study is that some arbitrary element $F$ of the anomalous gravitational field (disturbing potential, height of the geoid, anomaly of gravity, etc.) is arranged in a series by a small parameter characterizing the deviation of the reference ellipsoid from the sphere. The height of the geoid, the deflection is heavy, the anomaly of gravity and other similar elements of some function $F_{0}$ on the sphere are uniquely determined by the basic function of the disturbing potential $T_{0}$ by means of spherical relations. The corresponding functions $F$ will be the values of the elements on the ellipsoid and are also uniquely re-

${ }^{1}$ Ph.D., Associate Professor,

Lviv Polytechnic National University, Ukraine 
lated to $T=T_{0}$ by some ellipsoidal formulas. Accordingly, you can define functions $F_{1}$.

Based on the studies of the components of the Earth's anomalous gravitational field, we can summarize the following. The ellipsoidal correction $e^{2} \Delta g_{1}^{1}$ cannot be taken into account when calculating the Earth's force field, since the anomaly of gravity is almost independent of the offset of the reference coordinate system. But the anomaly of gravity strongly depends on the harmonics of the second degree, in particular, on the second zonal harmonic coefficient $C_{20}$, and so on the compression $f$ of the reference ellipsoid. The value of the ellipsoidal correction $e^{2} \Delta g_{2}^{1}$ obtained by us is of the same order of magnitude as the current high-precision gravimetric satellite data, so it must be taken into account when determining the Earth's force field. The results of the calculations performed in a spherical approximation showed that there is a strong dependence of the height of the geoid on the displacement of the reference coordinate system. Ellipsoidal corrections $e^{2} N_{1}^{1}, e^{2} N_{2}^{1}$ and $e^{2} N_{3}^{1}$ also need to be taken into account, since their values are of the same order of magnitude as modern high-precision altimeter-gravimetric calculations of the Earth's anomalous gravitational field.

Thus, the novelty and relevance of such scientific solutions lies in the expediency of taking into account ellipsoidal corrections in determining the anomalous terrestrial field of gravity, since the neglect of these corrections on the average for the territory of Ukraine gives an error in the accuracy of modern gravimetric satellite data and altimeter-gravimetric calculations of the Earth's anomalous gravitational field.

\section{Introduction}

Spherical approximation is the basis for almost all formulas of physical geodesy. It gives the error that is neglected in most practical applications. The problem of calculating the anomalous gravitational field or the perturbing potential of the Earth does not apply to this case, since the present gravimetric satellite data have now increased their accuracy by an order of magnitude in comparison with the recent past. Once such an error was an order of magnitude less than the accuracy provided by gravimetric satellite data of that time [3].

Therefore, it is logical to claim that ellipsoidal corrections should be taken into account in order to obtain higher accuracy in calculating the com- 
ponents of the anomalous terrestrial gravity field, as the difference between the values of the real and normal potential of the gravity force [7].

The methodology of such scientific research is that some arbitrary element $F$ of the anomalous gravitational field (disturbing potential, height of the geoid, anomaly of gravity, etc.) is arranged in a series by a small parameter $\varepsilon$ characterizing the deviation of the reference ellipsoid from the sphere [6]:

$$
F=F^{0}+\varepsilon F^{1}+\varepsilon^{2} F^{2}+\varepsilon^{3} F^{3}+\ldots .
$$

This parameter $\varepsilon$ can be compression $f$ or any other parameter of the ellipsoid, such as the square of the first eccentricity

$$
\varepsilon=e^{2}=\frac{a^{2}-b^{2}}{a^{2}} .
$$

Due to the small values of the above values, squares and higher degrees $\varepsilon$ can be neglected. As a result, this series can be written

$$
F=F^{0}+e^{2} F^{1} .
$$

Let us try to represent each element of an anomalous gravitational field in this form. It depends on the position of the point in space, which is expressed, for example, by geodetic coordinates $\phi$ (latitude) and $\lambda$ (longitude). In this case, this line can be written in a more concrete way

$$
F(\phi, \lambda)=F^{0}(\phi, \lambda)+e^{2} F^{1}(\phi, \lambda) .
$$

Since the function $F^{0}(\phi, \lambda)$ corresponds $e=0$, it can be considered as a function in a certain defined sphere, for example, in the middle earth sphere with radius

$$
R=\sqrt[3]{a^{2} b}=6371 \mathrm{~km} .
$$

Moreover, the coordinates $\phi$ and $\lambda$ are spherical coordinates in this sphere. Thus, it is possible to set a one-to-one mapping of the reference ellipsoid to a sphere with radius $R$ by designing a point with geodetic coordinates $(\phi, \lambda)$ on the ellipsoid to a point with spherical coordinates $(\phi, \lambda)$ on the sphere (values $\phi$ and $\lambda$ the same for both points).

Thus, the novelty and relevance of scientific solutions is the expediency of taking into account ellipsoidal corrections in determining the anomalies of gravity $\Delta g$ and heights of geoid $N$, since the neglect of these corrections on the average for the territory of Ukraine gives an erroneous order of accuracy for modern gravimetric satellite data and altimeter-gravimetric computations of the anomalous gravitational field of the Earth. 


\section{Disturbing potential}

The reflections described above connect the corresponding points on both surfaces. Now let us set the function display. Let the function $F^{0}(\phi, \lambda)$ on the sphere corresponds to the function $F(\phi, \lambda)$ on the ellipsoid and that both functions are related by relation (4).

Functions $F^{1}(\phi, \lambda)$ are defined as follows. Assuming that for disturbing potential [6]

$$
T^{1}(\phi, \lambda)=0,
$$

we get the basic mapping equation

$$
T^{0}(\phi, \lambda)=T(\phi, \lambda) .
$$

The height of the geoid, the weight line deviations, the anomaly of gravity and other similar elements $F^{0}(\phi, \lambda)$ in the sphere are uniquely determined through the main function $T^{0}(\phi, \lambda)$ by means of spherical relations. The corresponding functions $F(\phi, \lambda)$ will be the values of the elements on the ellipsoid and also uniquely related to the $T(\phi, \lambda)=T^{0}(\phi, \lambda)$ by some ellipsoidal formulas. Accordingly, functions $F^{1}(\phi, \lambda)$ can be defined.

Therefore, the content of the spherical approximation is to map the points of the ellipsoid with geodetic coordinates $(\phi, \lambda)$ to points on the sphere, the spherical coordinates of which are taken as coordinates $(\phi, \lambda)$ of the points on the ellipsoid. Moreover, the values of the first order $e^{2} F^{1}$ and the higher order terms are neglected [3].

It is necessary to find the same reflection by numerically comparing the geodetic coordinates on the ellipsoid and sphere, but with the preservation of the values of the first order.

It should be noted that the described transformation of geodetic coordinates into spherical ones is not the only way of establishing correspondence between the reference ellipsoid and the auxiliary sphere. You can also map the spherical coordinates to the ellipsoidal coordinates in the form of the given latitude $\beta$ and longitude $\lambda$. The use of $\beta$ and $\lambda$ leads to simpler formulas, but the use of $\phi$ and $\lambda$ has greater advantages from a practical point of view, since it is difficult to convert for many points geodetic coordinates $\phi$ into $\beta$ the above.

Note that M. Molodensky [5] transformed geocentric coordinates on an ellipsoid (geocentric latitude and longitude) into spherical coordinates when solving ellipsoidal boundary problems. 
Geographic (geodetic) coordinates were used by D. Lelgemann [11] and H. Moritz [6].

\section{Spherical functions}

In the study of the disturbing potential of the Earth, the method of decomposing the potential into a series of spherical functions is properly used. The same method of image potential proved to be quite convenient for studying the figure and the anomalous gravitational field of the Earth by the perturbations in motion of artificial satellites of the Earth [12;13].

This method makes it possible to represent the potential of gravity, which is given on a spherical Earth, in the form of the sum of harmonics, and the higher the harmonic order number, the smaller the wavelength. Defining the coefficients of such a trigonometric series is quite convenient for all kinds of calculations.

Researches to determine the size and shape of the Earth by the method of decomposition of gravitational potential into a series of spherical functions were dealt with in detail by B. Hoffmann-Wellenhof [1], P. Dvulit [2], D. Zagrebin [3], H. Moritz [6], Yu. Neuman, W. Heiskanen [10], D. Lelgemann [11].

The problem of the convergence of series by spherical functions is already a question of increased complexity. It is not elementary, as it seems at first glance, and has not been fully resolved yet from a theoretical point of view. The solution of this problem, which is suitable for practical purposes, is given by the well-known Runge's theorem [6;10].

That is, the series of spherical functions for the external gravitational potential is convergent for all points beyond some sphere $\sigma$, which is the smallest sphere into which the body $\tau$ can be inscribed.

Therefore, for the convergence of a series of spherical functions, it is sufficient that the point $P$, in which the potential $V$ is measured, lies outside the sphere $\sigma$. Although there are simple examples of convergent series on spherical functions, for which the convergence domain successfully extends into the middle of the sphere $\sigma[6 ; 10]$.

In this case, if the sphere $\sigma$ lies in the middle of some ellipsoid, then for an ellipsoid with a small compression (for example, as a compression of the Earth) it is safe to assume that the series is convergent over the entire surface of such ellipsoid. 
We denote by the smallest sphere $\sigma_{0}$ beyond which the external potential is regular, that is, it represents a harmonic function in the form of the Laplace's equation $\Delta V=0$. It is clear that such a sphere exists. Let us call it the boundary sphere. If the external potential cannot be analytically extended into the middle of the Earth, then the sphere $\sigma_{0}$ coincides with the sphere $\sigma$, otherwise, it lies inside the sphere $\sigma$. Therefore, the distribution of gravitational potential by spherical functions will be convergent at all points outside the sphere $\sigma_{0}$.

It is clear that the boundary sphere $\sigma_{0}$ can be defined as the smallest sphere, which has all the singularities of the analytic extension of the potential inside or out. It is an analogy with the circle of convergence in the theory of analytic functions of a complex variable. Such a circle of convergence divides the regions of convergence and divergence of a complex power series by analogy with the spatial row by spherical functions: convergence occurs everywhere within this circle, and divergence is everywhere beyond it $[6 ; 10]$.

On the basis of the above statements, we can assume that a similar situation applies to the three-dimensional case: the boundary sphere $\sigma_{0}$ (sphere of convergence) divides the regions of convergence outside the sphere $\sigma_{0}$ and the differences within the sphere $\sigma_{0}$.

Therefore, for spherical functions in space, the convergence surface is usually a sphere. Although there may be convergence surfaces other than the sphere, such as a cylinder or a torus. In particular, for zonal harmonics (Legendre polynomials) such a surface of convergence must be a sphere.

Thus, we see that the problem of convergence of a series of spherical functions of the external gravitational potential on the Earth's surface remains open for two reasons: 1) the properties of the analytic extension of the external potential into the middle of the Earth are not uniquely known; 2) the exact form of the convergence surface for series on spherical functions representing the Earth's potential is not known.

\section{Height of the geoid}

The height of the geoid $N$ is related to the disturbing potential $T$ ratio [6]

$$
N=\frac{T}{\gamma}
$$


where $\gamma$ is the normal gravity on the ellipsoid.

The approximate formula for calculating normal gravity $\gamma$ can be written $[6 ; 10]$

$$
\gamma=\gamma_{e}\left(1+f^{*} \sin ^{2} \phi\right)
$$

where $\gamma_{e}$ is the normal value of the acceleration of the gravity at the equator;

For the same approximation

$$
f^{*}=-f+\frac{5}{2} m \text {. }
$$

$$
f=\frac{1}{2} e^{2} .
$$

Numerical values [10] show that it is approximate

$$
m=\frac{1}{2} e^{2} .
$$

Therefore, formula (9) takes the form

$$
\gamma=\gamma_{e}\left(1+\frac{3}{4} e^{2} \sin ^{2} \phi\right) .
$$

Introducing Legendre polynomial

$$
P_{2}(\sin \phi)=\frac{3}{2} \sin ^{2} \phi-\frac{1}{2},
$$

we have

$$
\gamma=\gamma_{e}\left[1+\frac{1}{4} e^{2}+\frac{1}{2} e^{2} P_{2}(\sin \phi)\right] .
$$

Since the mean $P_{2}(\sin \phi)$ on the sphere is zero, the mean $\gamma$ on the sphere will be equal

$$
\gamma^{0}=\gamma_{e}\left(1+\frac{1}{4} e^{2}\right)
$$

so that formula (15) can be written as

$$
\gamma=\gamma^{0}\left[1+\frac{1}{2} e^{2} P_{2}(\sin \phi)\right]
$$

or taking into account the polynomial (14)

$$
\gamma=\gamma^{0}\left(1-\frac{1}{4} e^{2}+\frac{3}{4} e^{2} \sin ^{2} \phi\right) .
$$

Accordingly, relation (8) will have the form 


$$
N=\frac{T}{\gamma^{0}}\left(1+\frac{1}{4} e^{2}-\frac{3}{4} e^{2} \sin ^{2} \phi\right) .
$$

Given a constant spherical value $\gamma^{0}$ that corresponds to an ellipsoidal value $\gamma$, we obtain the value of the height of the geoid in the spherical approximation

So

$$
N^{0}=\frac{T^{0}}{\gamma^{0}}=\frac{T}{\gamma^{0}} \text {. }
$$

$$
N=N^{0}+e^{2} N^{1}
$$

where $N^{0}$ is the zero term of the decomposition that can be obtained from the spherical approximation; $e^{2} N^{1}$ is the so-called ellipsoidal correction or decomposition member characterizing the deviation of the reference ellipsoid from the sphere.

The magnitude $N^{0}$ can be calculated from the decomposition into a number of spherical functions $[4 ; 6 ; 10]$

$$
N^{0}=\frac{1}{\gamma} \sum_{n=0}^{\infty} \sum_{m=0}^{n}\left(A_{n m} \cos m \lambda+B_{n m} \sin m \lambda\right) P_{n m}(\sin \phi),
$$

where $\gamma$ is the normal value of the gravity.

The ellipsoidal term of a series $N^{1}$ can be related to a $N^{0}$ as follows [4]

$$
N^{1}=\left(\frac{1}{4}-\frac{3}{4} \sin ^{2} \phi\right) N^{0} .
$$

We before find the first term of the series (22). That is, for $n=1$ we obtain $[6 ; 10]$

$$
N_{1}^{0}=\frac{1}{\gamma}\left[A_{10} P_{10}(\sin \phi)+\left(A_{11} \cos \lambda+B_{11} \sin \lambda\right) P_{11}(\sin \phi)\right] .
$$

The coefficients $A_{10}, A_{11}$ and $B_{11}$ are related to the corresponding coefficients $C_{10}, C_{11}$ and $S_{11}$ by the following formulas [7]:

$$
A_{n m}=\frac{f M}{R} C_{n m} ; \quad B_{n m}=\frac{f M}{R} S_{n m} .
$$

It is known that the values $C_{10}, C_{11}$ and $S_{11}$ are related to the rectangular coordinates of the offset of the coordinate system by the following relations $[4 ; 8]$ :

$$
C_{10}=-\frac{\Delta z}{R} ; \quad C_{11}=-\frac{\Delta x}{R} ; \quad S_{11}=-\frac{\Delta y}{R} .
$$

Then, substituting (26) into (25), we obtain $[4,8]$

$$
A_{10}=-\frac{f M}{R^{2}} \Delta z ; \quad A_{11}=-\frac{f M}{R^{2}} \Delta x ; \quad B_{11}=-\frac{f M}{R^{2}} \Delta y .
$$


Using instead of $P_{10}(\sin \phi)$ and $P_{11}(\sin \phi)$ their values [7]

$$
P_{10}(\sin \phi)=\sin \phi ; \quad P_{11}(\sin \phi)=\cos \phi,
$$

given formula (27) and accepting $\frac{f M}{R^{2}}=\gamma$, the value $N_{1}^{0}$ will look like

$$
N_{1}^{0}=\Delta z \sin \phi+\Delta x \cos \lambda \cos \phi+\Delta y \sin \lambda \cos \phi .
$$

From formula (29) it is possible to notice the dependence of the heights of the geoid on the rectangular coordinates of the displacement of the system. To trace the numerical characteristic $N_{1}^{0}$, let us define the initial rectangular coordinates of the displacement of the parameters of the regional geodetic system (for example, the well-known European regional geodetic system European 1950) in the WGS 84 geocentric system $[6 ; 8]$

$$
\left.\begin{array}{rl}
\Delta a & =251 \mathrm{~m} ; \\
\Delta \alpha & =0.14192702 \times 10^{-4} ; \\
\Delta x & =-87 \mathrm{~m} ; \\
\Delta y & =-98 \mathrm{~m} ; \\
\Delta z & =-121 \mathrm{~m} .
\end{array}\right\}
$$

With the corresponding offset coordinates $(\Delta x, \Delta y, \Delta z)$, one can find the numerical characteristic of the spherical approximation $N_{1}^{0}$. To do this, select an $O$ point with spherical coordinates $\phi, \lambda$. It is quite appropriate to choose the center of the spheroidal trapezoid, which fits the territory of Ukraine, because it (the point) will be located near the geographical center of Ukraine with coordinates $\phi_{0}=48.3^{\circ}, \lambda_{0}=30.8^{\circ}$ [7].

Finally we get

$$
N_{1}^{0}=173.4 \mathrm{~m} \text {. }
$$

We now calculate the element of the ellipsoidal correction $N_{1}^{1}$. According to formula (23) for the above point $O\left(\phi_{0}, \lambda_{0}\right)$, this value can be written as follows:

$$
N_{1}^{1}=\left(\frac{1}{4}-\frac{3}{4} \sin ^{2} \phi_{0}\right) N_{1}^{0} .
$$

Substituting numerical values, we get

$$
N_{1}^{1}=-29.1 \mathrm{~m} \text {. }
$$

Then the entire ellipsoidal correction $e^{2} N_{1}^{1}$ will be equal 


$$
e^{2} N_{1}^{1}=-0.19 m
$$

Let us now investigate the term of the second degree of the decomposition of the height of the geoid $N^{0}$ into a series of spherical functions, that is, for $n=2$ the series (22) will be written

$$
\begin{aligned}
N_{2}^{0}=\frac{1}{\gamma}[ & A_{20} P_{20}(\sin \phi)+\left(A_{21} \cos \lambda+B_{21} \sin \lambda\right) P_{21}(\sin \phi)+ \\
& \left.+\left(A_{22} \cos 2 \lambda+B_{22} \sin 2 \lambda\right) P_{22}(\sin \phi)\right] .
\end{aligned}
$$

Considering that [7]

$P_{20}(\sin \phi)=\frac{1}{2}\left(3 \sin ^{2} \phi-1\right) ; P_{21}(\sin \phi)=3 \sin \phi \cos \phi ; P_{22}(\sin \phi)=3 \cos ^{2} \phi(36)$ and [7]

$$
\left.\begin{array}{l}
C_{20}=-1.082626 \times 10^{-3} ; \\
C_{21}=0 ; \\
C_{22}=1.571 \times 10^{-6} ; \quad S_{21}=0 ; \\
S_{22}=-9.03 \times 10^{-7}
\end{array}\right\}
$$

and finding by the formulas (25) the coefficients [7]

$$
\left.\begin{array}{ll}
A_{20}=-67734.3 \mathrm{~m}^{2} / \mathrm{s}^{2} ; & \\
A_{21}=0 ; & B_{21}=0 ; \\
A_{22}=98.3 \mathrm{~m}^{2} / \mathrm{s}^{2} ; & B_{22}=-56.5 \mathrm{~m}^{2} / \mathrm{s}^{2}
\end{array}\right\}
$$

for point $O$ with coordinates $\phi_{0}=48.3^{\circ}, \lambda_{0}=30.8^{\circ}$ the zero term of the schedule $N_{2}^{0}$ will be equal

$$
N_{2}^{0}=-2324.1 \mathrm{~m} \text {. }
$$

The results of (39) show a strong dependence of the height of the geoid, in particular, on the second zonal harmonic coefficient $C_{20}$.

Let us now research the ellipsoidal correction $e^{2} N_{2}^{1}$. Applying formula (23) to the above point $O\left(\phi_{0}, \lambda_{0}\right)$, we write first the value $N_{2}^{1}$. That is

$$
N_{2}^{1}=\left(\frac{1}{4}-\frac{3}{4} \sin ^{2} \phi_{0}\right) N_{2}^{0} .
$$

Substituting numerical values, we get

$$
N_{2}^{1}=390.7 \mathrm{~m} \text {. }
$$

Then the entire ellipsoidal correction $e^{2} N_{2}^{1}$ will be equal

$$
e^{2} N_{2}^{1}=2.6 \mathrm{~m} \text {. }
$$


Since the results of calculations (42) indicate that the ellipsoidal correction is large enough in absolute value $e^{2} N_{2}^{1}$, it is advisable to carry out further studies. We now find a member of the third degree of the decomposition of the height of the geoid $N^{0}$ into a series of spherical functions, when $n=3$, the series (22) is written

$$
N_{3}^{0}=\frac{1}{\gamma}\left[\begin{array}{l}
A_{30} P_{30}(\sin \phi)+\left(A_{31} \cos \lambda+B_{31} \sin \lambda\right) P_{31}(\sin \phi)+ \\
+\left(A_{32} \cos 2 \lambda+B_{32} \sin 2 \lambda\right) P_{32}(\sin \phi)+\left(A_{33} \cos 3 \lambda+B_{33} \sin 3 \lambda\right) P_{33}(\sin \phi)
\end{array}\right] .
$$

Considering that $[6,10]$

$$
\left.\begin{array}{l}
P_{30}(\sin \phi)=\frac{1}{2}\left(5 \sin ^{3} \phi-3 \sin \phi\right) ; P_{31}(\sin \phi)=-\frac{3}{2}\left(5 \sin ^{2} \phi-1\right)\left(1-\sin ^{2} \phi\right)^{1 / 2} ;
\end{array}\right\}
$$

and finding by (25) the coefficients

$$
\left.\begin{array}{ll}
A_{30}=158.6 \mathrm{~m}^{2} / \mathrm{s}^{2} ; & \\
A_{31}=137.1 \mathrm{~m}^{2} / \mathrm{s}^{2} ; & B_{31}=17.0 \mathrm{~m}^{2} / \mathrm{s}^{2} ; \\
A_{32}=55.9 \mathrm{~m}^{2} / \mathrm{s}^{2} ; & B_{32}=-39.0 \mathrm{~m}^{2} / \mathrm{s}^{2} ; \\
A_{33}=43.8 \mathrm{~m}^{2} / \mathrm{s}^{2} ; & B_{33}=88.3 \mathrm{~m}^{2} / \mathrm{s}^{2},
\end{array}\right\}
$$

for point $O$ with coordinates $\phi_{0}=48.3^{\circ}, \lambda_{0}=30.8^{\circ}$ the zero term of the schedule $N_{3}^{0}$ will be equal

$$
N_{3}^{0}=-67.0 \mathrm{~m} .
$$

Let us now investigate the ellipsoidal correction $e^{2} N_{3}^{1}$. Applying formula (23) to the above point $O\left(\phi_{0}, \lambda_{0}\right)$, we write first the value $N_{3}^{1}$. That is

$$
N_{3}^{1}=\left(\frac{1}{4}-\frac{3}{4} \sin ^{2} \phi_{0}\right) N_{3}^{0} .
$$

Substituting numerical values, we get

$$
N_{3}^{1}=11.3 \mathrm{~m} \text {. }
$$

Then the entire ellipsoidal correction $e^{2} N_{3}^{1}$ will be equal

$$
e^{2} N_{3}^{1}=0.076 m=7.6 \mathrm{~cm} \text {. }
$$


From the results of calculations (50) it is safe to say: the error for the heights of the geoid $N$ due to neglect of the ellipsoidal correction $e^{2} N_{3}^{1}$ on the average for the territory of Ukraine gives a value of \pm 7.6 $\mathrm{cm}$, which has the same order of accuracy, which is provided by modern altimeter-gravimetric calculations of the anomalous gravitational field of the Earth.

\section{Weight line deviations}

The components of the deviation are severely determined by the formulas [6]

$$
\xi=-\frac{\partial N}{\partial s_{\phi}} ; \quad \eta=-\frac{\partial N}{\partial s_{\lambda}},
$$

where the differentiation of the height of the geoid $N$ is carried out along the meridian and along the parallel respectively. The full differential of arc length on the rotation ellipsoid has the form $[6 ; 10]$

$$
d s^{2}=\mu^{2} d \phi^{2}+v^{2} \cos ^{2} \phi d \lambda^{2},
$$

where $\propto$ and $v$ are the principal radii of curvature of an ellipsoid, which are defined by formulas

$$
\begin{aligned}
\mu & =\frac{c}{V^{3}}, \\
\nu & =\frac{c}{V} .
\end{aligned}
$$

The designation here is accepted

$$
V=\left(1+e^{\prime 2} \cos ^{2} \phi\right)^{\frac{1}{2}}
$$

and the formulas used $[6 ; 10]$

$$
\begin{gathered}
c=\frac{a^{2}}{b}, \\
e^{\prime 2}=\frac{e^{2}}{1-e^{2}},
\end{gathered}
$$

where $c$ is the polar radius of curvature; $e^{\prime 2}$ is the second eccentricity. Since [10]

$$
\left.\begin{array}{l}
d s_{\phi}=\mu d \phi ; \\
d s \lambda=v \cos \phi d \lambda,
\end{array}\right\}
$$

then expressions (51) can be rewritten as 


$$
\xi=-\frac{1}{\mu} \frac{\partial N}{\partial \phi} ; \quad \eta=-\frac{1}{v \cos \phi} \frac{\partial N}{\partial \lambda} .
$$

In spherical approximation

$$
\xi^{0}=-\frac{1}{R} \frac{\partial N^{0}}{\partial \phi} ; \quad \eta^{0}=-\frac{1}{R \cos \phi} \frac{\partial N^{0}}{\partial \lambda} .
$$

We take advantage of the series in which members are held in order $e^{2}$, that is

$$
\begin{gathered}
R=\sqrt[3]{a^{2} b}=a\left(1-\frac{1}{6} e^{2}\right), \\
c=a^{2} b=a\left(1+\frac{1}{2} e^{2}\right)=R\left(1+\frac{2}{3} e^{2}\right), \\
V=1+\frac{1}{2} e^{2} \cos ^{2} \phi=1+\frac{1}{2} e^{2}-\frac{1}{2} e^{2} \sin ^{2} \phi, \\
\frac{1}{\mu}=\frac{1}{R}\left(1+\frac{5}{6} e^{2}-\frac{3}{2} e^{2} \sin ^{2} \phi\right), \\
\frac{1}{v}=\frac{1}{R}\left(1-\frac{1}{6} e^{2}-\frac{1}{2} e^{2} \sin ^{2} \phi\right) .
\end{gathered}
$$

Further, according to expressions (21) and (23), we obtain

$$
\frac{\partial N}{\partial \phi}=\frac{\partial N^{0}}{\partial \phi}+e^{2} \frac{\partial N^{1}}{\partial \phi}=\frac{\partial N^{0}}{\partial \phi}+e^{2}\left(\frac{1}{4}-\frac{3}{4} \sin ^{2} \phi\right) \frac{\partial N^{0}}{\partial \phi}-e^{2} \frac{3}{2} \sin \phi \cos \phi N^{0}
$$

and similarly

$$
\frac{\partial N}{\partial \lambda}=\frac{\partial N^{0}}{\partial \lambda}+e^{2}\left(\frac{1}{4}-\frac{3}{4} \sin ^{2} \phi\right) \frac{\partial N^{0}}{\partial \lambda} .
$$

Substituting these expressions together with expressions (64) and (65) in (59) and considering formulas (60), we finally obtain

where

$$
\left.\begin{array}{l}
\xi=\xi^{0}+e^{2} \xi^{1} ; \\
\eta=\eta^{0}+e^{2} \eta^{1},
\end{array}\right\}
$$

$$
\begin{gathered}
\xi^{1}=\left(\frac{13}{12}-\frac{9}{4} \sin ^{2} \phi\right) \xi^{0}+\frac{3}{2} \sin \phi \cos \phi \frac{N^{0}}{R}, \\
\eta^{1}=\left(\frac{1}{12}-\frac{5}{4} \sin ^{2} \phi\right) \eta^{0} .
\end{gathered}
$$

Since the quantities $e^{2} \xi^{1}$ and $e^{2} \eta^{1}$ are very small, then for $\xi^{0}, \eta^{0}, N^{0}$, 
which are necessary for the calculation $\xi^{1}$ and $\eta^{1}$, any approximate values can be used, for example, obtained from a finite arrangement of spherical functions.

\section{Anomalies of gravity}

The anomaly of gravity $\Delta g$ can be represented in the form suggested by Moritz in [6] or [10], that is

$$
\Delta g=\Delta g^{0}+e^{2} \Delta g^{1}
$$

where

$$
\begin{gathered}
\Delta g^{0}=\sum_{n=0}^{\infty} \sum_{m=0}^{n} \frac{n-1}{R} P_{n m}(\sin \phi)\left(A_{n m} \cos m \lambda+B_{n m} \sin m \lambda\right) \\
\Delta g^{1}=\frac{1}{R} \sum_{n=0}^{\infty} \sum_{m=0}^{n} P_{n m}(\sin \phi)\left(G_{n m} \cos m \lambda+H_{n m} \sin m \lambda\right)
\end{gathered}
$$

with

$$
\left.\begin{array}{c}
G_{n m}=\chi_{n+2, m} A_{n m}+\lambda_{n+2, m} A_{n+2, m}+\mu_{n+2, m} A_{n+4, m} \\
H_{n m}=\chi_{n+2, m} B_{n m}+\lambda_{n+2, m} B_{n+2, m}+\mu_{n+2, m} B_{n+4, m}
\end{array}\right\}
$$

and

$$
\left.\begin{array}{l}
\chi_{n m}=-\frac{3(n-3)(n-m-1)(n-m)}{2(2 n-3)(2 n-1)} ; \\
\lambda_{n m}=\frac{n^{3}-3 m^{2} n-9 n^{2}-6 m^{2}-10 n+9}{3(2 n+3)(2 n-1)} ; \\
\mu_{n m}=-\frac{(3 n+5)(n+m+2)(n+m+1)}{2(2 n+5)(2 n+3)} .
\end{array}\right\}
$$

Let us write down the first member of the schedule $\Delta g$, that is [7]

$$
\Delta g_{1}=\Delta g_{1}^{0}+e^{2} \Delta g_{1}^{1} .
$$

It is easy to note from formula (72) that since $n=1$, then

$$
\Delta g_{1}^{0}=0 \text {. }
$$

Since the spherical approximation $\Delta g_{1}^{0}$ is zero, we should investigate the element of the ellipsoidal correction $\Delta g_{1}^{1}$ that is written [7]

$$
\Delta g_{1}^{1}=\frac{1}{R}\left[P_{10}(\sin \phi) G_{10}+P_{11}(\sin \phi)\left(G_{11} \cos \lambda+H_{11} \sin \lambda\right)\right] .
$$

It is known $[4 ; 7]$ that

$$
P_{10}(\sin \phi)=\sin \phi ; \quad P_{11}(\sin \phi)=\cos \phi,
$$

Substituting the corresponding values from expression (75) into formula (74), we obtain $[6 ; 7]$ 


$$
\left.\begin{array}{l}
G_{10}=\chi_{30} A_{10}+\lambda_{30} A_{30}+\mu_{30} A_{50} ; \\
G_{11}=\chi_{31} A_{11}+\lambda_{31} A_{31}+\mu_{31} A_{51} ; \\
H_{11}=\chi_{31} B_{11}+\lambda_{31} B_{31}+\mu_{31} B_{51}
\end{array}\right\}
$$

Having formulas (78) - (80), one can find the numerical characteristic of the ellipsoidal correction element $\Delta g_{1}^{1}$. To do this, select a point $O$ with spherical coordinates $\phi, \lambda$ (see formula (78)). It is quite appropriate for point $O(\phi, \lambda)$ to choose the center of the spheroidal trapezoid, which fits the territory of Ukraine, since it (point) will be located near the geographical center of Ukraine with coordinates $\phi_{0}=48.3^{\circ}, \lambda_{0}=30.8^{\circ}$ [7].

For the basic fundamental parameters of the Earth take the following values $[1 ; 2 ; 9]$ :

$$
R=6371000 \mathrm{~m} ; f M=398600.5 \times 10^{9} \mathrm{~m}^{3} / \mathrm{s}^{2} .
$$

Dimensionless harmonic coefficients $C_{n m}$ and $S_{n m}$ (for example, the GEM 10 model) have the following values $[12,13]$ :

$$
\left.\begin{array}{l}
C_{30}=2.535 \times 10^{-6} ; \quad C_{50}=2.29 \times 10^{-7} ; \\
C_{31}=2.192 \times 10^{-6} ; \quad C_{51}=-4.4 \times 10^{-8} ; \\
S_{31}=2.72 \times 10^{-7} ; \quad S_{51}=-8.0 \times 10^{-8} .
\end{array}\right\}
$$

Then the values of formulas (75) will be equal [7]

$$
\left.\begin{array}{lll}
\chi_{30}=0 ; & \lambda_{30}=-\frac{5}{9} ; & \mu_{30}=-\frac{140}{99} ; \\
\chi_{31}=0 ; & \lambda_{31}=-\frac{29}{45} ; & \mu_{31}=-\frac{70}{33}
\end{array}\right\}
$$

Since it is known $[9 ; 11]$ that

then [7]

$$
A_{n m}=\frac{f M}{R} C_{n m} ; \quad B_{n m}=\frac{f M}{R} S_{n m},
$$

$$
\left.\begin{array}{ll}
A_{30}=158.6 \mathrm{~m}^{2} / \mathrm{s}^{2} ; & A_{50}=14.3 \mathrm{~m}^{2} / \mathrm{s}^{2} ; \\
A_{31}=137.1 \mathrm{~m}^{2} / \mathrm{s}^{2} ; & A_{51}=-2.7 \mathrm{~m}^{2} / \mathrm{s}^{2} ; \\
\boldsymbol{B}_{31}=17.0 \mathrm{~m}^{2} / \mathrm{s}^{2} ; & \boldsymbol{B}_{51}=-5.0 \mathrm{~m}^{2} / \mathrm{s}^{2} .
\end{array}\right\}
$$

Substituting these values into formula (80), we obtain [7]

$$
\left.\begin{array}{l}
G_{10}=-108.3 \mathrm{~m}^{2} / \mathrm{s}^{2} ; \\
G_{11}=-82.6 \mathrm{~m}^{2} / \mathrm{s}^{2} ; \\
H_{11}=-0.3 \mathrm{~m}^{2} / \mathrm{s}^{2} .
\end{array}\right\}
$$


Then, substituting the above values into formula (78), we find [7]

or [7]

$$
\Delta g_{1}^{1}=-2.0 \times 10^{-5} \mathrm{~m} / \mathrm{s}^{2}
$$

$$
\Delta g_{1}^{1}=-2.0 \mathrm{mGal} \text {. }
$$

The value of $\Delta g_{1}$ (see formula (6)) will then be equal [7]

$$
\Delta g_{1}=-0.01 \mathrm{mGal} .
$$

It is well known that a member of the first degree $\Delta g_{1}$ of decomposition of anomalies of gravity $\Delta g$, as transformants of perturbing gravitational potential, in a number of spherical functions indicates a shift of the coordinate system (see, for example, works $[4 ; 5 ; 8]$ ). That is, the linear term $\Delta g_{1}$ causes an effect on the anomaly of gravity due to the displacement of the reference coordinate system.

Now let us consider the influence of a member of the second degree $\Delta g_{2}$ of decomposition into a number of spherical functions, taking into account its ellipsoidal correction on the anomaly of gravity. Then formula (71) is written [7]

$$
\Delta g_{2}=\Delta g_{2}^{0}+e^{2} \Delta g_{2}^{1},
$$

where

$$
\begin{gathered}
\Delta g_{2}^{0}=\frac{1}{R}\left[P_{20}(\sin \phi) A_{20}+P_{21}(\sin \phi)\left(A_{21} \cos \lambda+B_{21} \sin \lambda\right)+\right. \\
\left.+P_{22}(\sin \phi)\left(A_{22} \cos 2 \lambda+B_{22} \sin 2 \lambda\right)\right] ; \\
\Delta g_{2}^{1}=\frac{1}{R}\left[P_{20}(\sin \phi) G_{20}+P_{21}(\sin \phi)\left(G_{21} \cos \lambda+H_{21} \sin \lambda\right)+\right. \\
\left.+P_{22}(\sin \phi)\left(G_{22} \cos 2 \lambda+H_{22} \sin 2 \lambda\right)\right] .
\end{gathered}
$$

Believing that $[7,9]$

$$
\begin{aligned}
& P_{20}(\sin \phi)=\frac{1}{2}\left(3 \sin ^{2} \phi-1\right) ; P_{21}(\sin \phi)=3 \sin \phi \cos \phi ; P_{22}(\sin \phi)=3 \cos ^{2} \phi \\
& \text { and [7; 9] } \\
& \left.\begin{array}{rl}
C_{20} & =-1.082626 \times 10^{-3} ; \\
C_{21} & =0 ; \\
C_{22} & =1.571 \times 10^{-6} ; \quad S_{22}=0 ;
\end{array}\right\}
\end{aligned}
$$

and having found by the formulas (84) the coefficients [7]

$$
\left.\begin{array}{ll}
A_{20}=-67734.3 \mathrm{~m}^{2} / \mathrm{s}^{2} ; & \\
A_{21}=0 ; & B_{21}=0 ; \\
A_{22}=98.3 \mathrm{~m}^{2} / \mathrm{s}^{2} ; & B_{22}=-56.5 \mathrm{~m}^{2} / \mathrm{s}^{2}
\end{array}\right\}
$$


for point $O$ with coordinates $\phi_{0}=48.3^{\circ}$ and $\lambda_{0}=30.8^{\circ}$, we compute the zero term of the schedule $\Delta g_{2}^{0}$. It will be equal [7]

$$
\Delta g_{2}^{0}=-350 \mathrm{mGal} \text {. }
$$

The results of (96) show a strong dependence of the anomaly of gravity, in particular, on the second zonal harmonic coefficient $C_{20}$.

Let us now investigate the ellipsoidal correction $e^{2} \Delta g_{2}^{1}$. We first find the coefficients $G_{n m}$ and $H_{n m}$ those contained in formula (92), that is [7]

$$
\left.\begin{array}{ll}
G_{20}=\chi_{40} A_{20}+\lambda_{40} A_{40}+\mu_{40} A_{60} ; & \\
G_{21}=\chi_{41} A_{21}+\lambda_{41} A_{41}+\mu_{41} A_{61} ; & H_{21}=\chi_{41} B_{21}+\lambda_{41} B_{41}+\mu_{41} B_{61} ; \\
G_{22}=\chi_{42} A_{22}+\lambda_{42} A_{42}+\mu_{42} A_{62} ; & H_{22}=\chi_{42} B_{22}+\lambda_{42} B_{42}+\mu_{42} B_{62} .
\end{array}\right\}
$$

The corresponding harmonic coefficients $C_{n m}$ and $S_{n m}$ have the following meanings [7]:

$$
\left.\begin{array}{ll}
C_{40}=5.39 \times 10^{-7} ; & C_{60}=-1.48 \times 10^{-7} ; \\
C_{41}=-5.33 \times 10^{-7} ; & C_{61}=-8.1 \times 10^{-8} ; \\
C_{42}=3.47 \times 10^{-7} ; & C_{62}=5.2 \times 10^{-8} ; \\
S_{41}=-4.75 \times 10^{-7} ; & S_{61}=2.4 \times 10^{-8} ; \\
S_{42}=6.64 \times 10^{-7} ; & S_{62}=-3.75 \times 10^{-7}
\end{array}\right\}
$$

and we take the values of coefficients $C_{20}, C_{21}, C_{22}$ and $S_{21}, S_{22}$ from formulas (94).

Then the values of formulas (75) after the calculations will be equal [7]

$$
\left.\begin{array}{lll}
\chi_{40}=\frac{36}{70} ; & \lambda_{40}=-\frac{111}{231} ; & \mu_{40}=\frac{510}{286} ; \\
\chi_{41}=\frac{18}{70} ; & \lambda_{41}=-\frac{129}{231} ; & \mu_{41}=\frac{714}{286} ; \\
\chi_{42}=\frac{6}{70} ; & \lambda_{42}=-\frac{183}{231} ; & \mu_{42}=\frac{952}{286}
\end{array}\right\}
$$

Using formulas (84), we calculate the coefficients $A_{n m}$ and $B_{n m}$ with the corresponding indices, we obtain [7]

$$
\left.\begin{array}{l}
A_{40}=33.7 \mathrm{~m}^{2} / \mathrm{s}^{2} ; \quad A_{60}=-9.2 \mathrm{~m}^{2} / \mathrm{s}^{2} ; \\
A_{41}=-33.3 \mathrm{~m}^{2} / \mathrm{s}^{2} ; \quad A_{61}=-5.1 \mathrm{~m}^{2} / \mathrm{s}^{2} ; \\
A_{42}=21.7 \mathrm{~m}^{2} / \mathrm{s}^{2} ; \quad A_{62}=3.2 \mathrm{~m}^{2} / \mathrm{s}^{2} ; \\
B_{41}=-29.7 \mathrm{~m}^{2} / \mathrm{s}^{2} ; \quad B_{61}=1.5 \mathrm{~m}^{2} / \mathrm{s}^{2} ; \\
B_{42}=41.5 \mathrm{~m}^{2} / \mathrm{s}^{2} ; \quad B_{62}=-23.5 \mathrm{~m}^{2} / \mathrm{s}^{2}
\end{array}\right\}
$$


and we take the values of coefficients $A_{20}, A_{21}, A_{22}$ and $B_{21}, B_{22}$ from formulas (95).

Substituting these values into formula (97), we obtain [7]

$$
\left.\begin{array}{l}
G_{20}=-34867.4 \mathrm{~m}^{2} / \mathrm{s}^{2} ; \\
G_{21}=5.9 \mathrm{~m}^{2} / \mathrm{s}^{2} ; \quad H_{21}=20.3 \mathrm{~m}^{2} / \mathrm{s}^{2} ; \\
G_{22}=1.9 \mathrm{~m}^{2} / \mathrm{s}^{2} ; \quad H_{22}=-115.9 \mathrm{~m}^{2} / \mathrm{s}^{2} .
\end{array}\right\}
$$

Using formulas (92), (93) and the results of calculations (101), for a point $O$ with coordinates $\phi_{0}=48.3^{\circ}$ and $\lambda_{0}=30.8^{\circ}$, we find the numerical characteristic of the ellipsoidal correction element $\Delta g_{2}^{1}$. Obtain the value [7]

or [7]

$$
\Delta g_{2}^{1}=-1.857 \times 10^{-3} \mathrm{~m} / \mathrm{s}^{2}
$$

$$
\Delta g_{2}^{1}=-185.7 \mathrm{mGal} .
$$

Then all the ellipsoidal correction $e^{2} \Delta g_{2}^{1}$ according to formula (90) will take the following value [7]:

$$
e^{2} \Delta g_{2}^{1}=-1.24 \mathrm{mGal} \text {. }
$$

From the results of calculations (104) it is safe to say that the error for anomalies of gravity $\Delta g$ due to neglect of the ellipsoidal correction $e^{2} \Delta g_{2}^{1}$ on the average for the territory of Ukraine gives a value of $\pm 1.24 \mathrm{mGal}$, which has the same order of accuracy as is provided by modern gravimetric satellite data.

\section{Conclusions}

Thus, based on the performed studies of the components of the Earth's anomalous gravitational field, we can summarize the following:

1. There is a strong dependence of the height of the geoid on the displacement of the reference coordinate system, which is shown by the results of calculations in a spherical approximation (31).

2. With respect to ellipsoidal corrections $e^{2} N_{1}^{1}, e^{2} N_{2}^{1}$ and $e^{2} N_{3}^{1}$, they should also be taken into account, since their values, presented in the form of results (34), (42) and (50), respectively, have values of the same order as modern high-precision altimeter-gravimetric calculations of the Earth's anomalous gravitational field.

3 . The gravity anomaly $\Delta g$ is almost independent of the offset of the reference coordinate system. This is clearly demonstrated by (77) and (89), so 
ellipsoidal correction $e^{2} \Delta g_{1}^{1}$ cannot be taken into account when calculating the Earth's anomalous gravitational field.

4. The gravity anomaly depends strongly on the second-degree harmonics, in particular, on the second zonal harmonic coefficient $C_{20}$, and therefore on the compression $f$ of the reference ellipsoid.

5. With respect to ellipsoidal correction $e^{2} \Delta g_{2}^{1}$, it must be taken into account, since its value, represented in the form of results (104), is of the same order of magnitude as the current high-precision gravimetric satellite data.

\section{References:}

1. Hoffmann-Wellenhof B., Moritz H. (2007). Fizicheskaya geodeziya [Physical geodesy]. Moscow. (in Russian)

2. Dvulit P.D. (2008). Fizychna Heodeziia [Physical geodesy]. Kyiv. (in Ukrainian)

3. Zagrebin D.V. (1952). Teoriya regulyarizirovannogo geoida [Theory of regularized geoid]. Trudy ITA, no. 1, pp. 52-61.

4. Meshcheryakov G.A., TSerklevich A.L. (1987). Gravitatsionnoye pole, figura $i$ vnutrenneye stroyeniye Marsa [Gravitational field, figure and internal structure of Mars]. Kiyev. (in Russian)

5. Molodenskiy M.S., Eremeyev V.F., YUrkina M.I. (1960). Metody izucheniya vneshnego gravitatsionnogo polya i figury Zemli. [Methods for studying the external gravitational field and the figure of the Earth]. Trudy TSNIIGAiK, vol. 131, pp. 250-251.

6. Moritz H. (1983). Sovremennaya fizicheskaya geodeziya [Advanced physical geodesy]. Moscow. (in Russian)

7. Sohor A.R., Sohor M.A. (2019). Pro vplyv elipsoidalnykh popravok na vyznachennia anomalii syly vahy [On the influence of ellipsoidal corrections on the determination of anomalies of gravity]. Molodyi vchenyi, no. 8 (72), pp. 153-156.

8. Tikhonov A.N., Samarskiy A.A. (1966). Uravneniya matematicheskoy fiziki [Equations of mathematical physics]. Moscow. (in Russian)

9. Boucher C., Altamimi Z. (2001). ITRS, PZ-90 and WGS-84: Current Realizations and the Related Transformation Parameters. Journal of Geodesy, vol. 75, pp. 613-619.

10. Heiskanen W. and Moritz H. (1967). Physical Geodesy. W.H. Freeman and Company. San Francisco, California.

11. Lelgemann D. (1973). Spherical Approximation and the Combination of Gravimetric and Satellite Data. Boll. Geold. Sci. Affini, vol. 32, pp. 241-250.

12. Lemoine J., Bourgogne S., Biancal R., Reinquin F., Bruinsma S. (2019) EIGEN-GRGS.RL04.MEAN-FIELD. Model of the Earth's Gravitational Field with Time Variable Part CNES/GRGS RL04. Available at: https://grace.obs-mip.fr/ variable-models-grace-lageos/mean-fields/release-04 (accessed 12 August 2019).

13. Zingerle P., Brockmann J., Pail R., Gruber T., Willberg M. (2019) GO CONS GCF-2 TIM R6e. Polar Model of Extended Gravitational Field TIM_R6. DOI: 10.5880/ICGEM.2019.005. 